\title{
Graphene Andreev Billiards
}

\author{
József Cserti and Imre Hagymási \\ Department of Physics of Complex Systems, Eötvös University, \\ H-1117 Budapest, Pázmány Péter sétány 1/A, Hungary \\ Andor Kormányos \\ Department of Physics, Lancaster University, \\ Lancaster, LA1 $4 Y B, U K$
}

\begin{abstract}
We studied the energy levels of graphene based Andreev billiards consisting of a superconductor region on top of a monolayer graphene sheet. For the case of Andreev retro-reflection we show that the graphene based Andreev billiard can be mapped to the normal metal-superconducting billiards with the same geometry. We also derived a semiclassical quantization rule in graphene based Andreev billiards. The exact and the semiclassically obtained spectrum agree very well both for the case of Andreev retro-reflection and specular Andreev reflection.

PACS numbers: 74.45.+c,74.50.+r,74.78.Na,03.65.Sq
\end{abstract}

In the well-known Andreev billiards consisting of a normal metal surrounded by a superconductor (NS) the dynamics of the quasiparticles is determined by the socalled Andreev retro-reflection [1]. The spectrum of Andreev billiards is described by the Bogoliubov-de Gennes (BdG) equation and has been long studied [2, 3] (for review of the topic see, eg, [4]).

The electronic properties of graphene can be described accurately by massless Dirac fermion type excitations using two dimensional relativistic quantum mechanics [5, 6, 7] and also by semiclassical methods [8] (for reviews on the physics of graphene see, e.g., [9]). In the seminal paper by Beenakker [10] it has been shown that when monolayer graphene is interfaced with a superconductor then two types of Andreev reflection are possible depending on the ratio of the Fermi energy $E_{\mathrm{F}}^{(\mathrm{G})}$ and the electron energy $E$. For $E_{\mathrm{F}}^{(\mathrm{G})}>\Delta^{(G)}>E$ the Andreev retro-reflection is dominant as in NS billiards (here $\Delta^{(G)}$ is the superconducting pair potential induced in the graphene). In contrast, when $E_{\mathrm{F}}^{(\mathrm{G})}<E<\Delta^{(G)}$, a different type of scattering process takes place at the graphene-superconductor interface, which is named specular Andreev reflection. The specular Andreev reflection does not exist in NS systems and it is a prominent consequence of the peculiar band structure of the monolayer graphene. Beenakker's paper has been followed by numerous works 11] (for a review on Andreev reflection in graphene see article [12]). Note that although graphene itself is not superconducting, due to the proximity effect a superconductor can induce non-zero pair-potential in the graphene as well. Indeed, supercurrent has been observed experimentally [13] between two superconducting electrodes on top of a graphene monolayer. Moreover, experimental results of Ref. [14] attest to the ballistic propagation of quasiparticles in graphene-superconductor hybrid structures.

The most widely studied theoretical model of Andreev billiards is that of a two dimensional electron gas (2DEG) in a quantum dot contacted by a bulk superconductor (see eg. [4]). One of the major obstacles that has thwarted so far the direct comparison of the theoretical predictions and experimental results is the inevitably existing tunnel barrier and mismatch of the Fermi-velocities and effective masses between the 2DEG and the superconductor (often referred to as "non-ideal NS interface" in the literature). This mismatch causes the probability of normal reflection to increase at the NS interface while the probability of the Andreev reflection diminishes significantly. The situation when both normal and Andreev reflection take place at the NS interface is theoretically more difficult to address. In graphene however, when the superconductivity is induced by external superconducting contacts, such mismatch may not exist so that the graphene-superconductor systems may experimentally be ideal to study most of the theoretical predictions made assuming perfect (ie with no mismatch) NS-interfaces.

In this paper we consider graphene Andreev billiards (GABs). In particular, we assume that in a closed region $\mathcal{D}$ of the graphene sheet the superconducting pair potential is zero and outside this region it takes on a constant value $\Delta^{(G)}$. We demonstrate, in one hand, that when the retro-reflection is the dominant scattering process at the normal graphene-superconductor interface the electronic properties of GABs can indeed be obtained in semiclassical approximation from the known results for NS billiards with ideal NS interface. On the other hand, we also calculate the exact spectrum of a GAB for the case when the dominant scattering process is the specular Andreev reflection and we show that it can also be understood using semiclassical considerations.

To see the relation between the energy spectrum of NS billiards and GABs note the following: the dispersion relation of the quasiparticles in the normal $\left(\Delta^{(N)}=0\right)$ region of the NS billiards for energies $E<\Delta^{(N)}$ can be linearized around the Fermi energy $E_{\mathrm{F}}^{(\mathrm{N})}$ as $E(p)=$ 
$\pm v_{\mathrm{F}}^{(N)}\left(p-p_{\mathrm{F}}^{(N)}\right)$, where the sign $+(-)$ refers to the electron-like (hole-like) quasiparticles. Here $p$ is the magnitude of the momentum, $p_{\mathrm{F}}^{(N)}=\sqrt{2 m E_{\mathrm{F}}^{(\mathrm{N})}}$ is the Fermi momentum and $v_{\mathrm{F}}^{(N)}=p_{\mathrm{F}}^{(N)} / m$ is the the Fermi velocity. This linearization is allowed if we are interested in the properties of the bound states $\left(E<\Delta^{(N)}\right)$ of NS billiards because for typical NS billiards the dimensionless parameter $\Delta^{(N)} / E_{F}^{(N)} \ll 1$ is much less than unity. The same linear dispersion can be found for electronlike (hole-like) quasiparticles in the $\Delta^{(G)}=0$ region for GABs in the retro-reflection regime but with Fermi velocity $v_{\mathrm{F}}^{(G)}$ and Fermi momentum $p_{\mathrm{F}}^{(G)}$. This simple observation is the core of the intimate relation between the graphene based and normal metal Andreev billiards. As long as the effect of the superconductor in semiclassical approximation can be described by the same way for GABs as for the NS billiards, i.e. by a simple phase shift $-\arccos \left(E / \Delta^{(N, G)}\right)$, one can expect that when the Andreev retro-reflection is the dominant scattering process the gross features of the energy spectrum of a GAB will closely resemble the spectrum of a NS billiard having the same geometry. This happens because the quasiparticles have linear dispersion in both cases. Moreover, if the Fermi velocities and Fermi momentums are the same i.e., $v_{\mathrm{F}}=\hbar k_{\mathrm{F}}^{(N)} / m=E_{\mathrm{F}}^{(\mathrm{G})} /\left(\hbar k_{\mathrm{F}}^{(G)}\right)$ and $p_{\mathrm{F}}=\sqrt{2 m E_{\mathrm{F}}^{(N)}}=E_{\mathrm{F}}^{(G)} / v_{\mathrm{F}}$ the quasiparticles in the $\Delta^{(N, G)}=0$ region will have the same dispersion relation for both NS billiards and GABs. Note that if $p_{\mathrm{F}}$ and $v_{\mathrm{F}}$ are the same then $E_{\mathrm{F}}^{(N)}=E_{\mathrm{F}}^{(G)} / 2$.

To demonstrate the idea discussed above we consider a simple, circular shape GAB. It consists of normal graphene region of radius $R$ surrounded by superconducting graphene. Owing to the valley degeneracy of the Hamiltonian the full BdG equation for graphenesuperconductor systems decouples to two four by four, reduced Hamiltonians that are related to each other by a unitary transformation (see, e.g., [10]). We now take the one corresponding to the valley $\mathbf{K}$. Due to the circular symmetry of the setup the reduced Hamiltonian is separable in polar coordinates and therefore the eigenfunctions can be labelled by an integer number $m$ corresponding to the angular momentum quantum number. One can show that the ansatz for the wave functions satisfying the Schrödinger equation for the reduced Hamiltonian in the region where $\Delta^{(G)}=0$, ie, for $r<R$ with energy $E$ are $\Psi_{m}^{(\mathrm{N})}(r, \varphi)=\left(c_{+}^{(\mathrm{N})} \chi_{+}^{(\mathrm{N})}(r, \varphi)+c_{-}^{(\mathrm{N})} \chi_{-}^{(\mathrm{N})}(r, \varphi)\right) e^{i m \varphi}$, where $\chi_{+}^{(\mathrm{N})}(r, \varphi)=\left[-i J_{m}\left(k_{+} r\right), J_{m+1}\left(k_{+} r\right) e^{i \varphi}, 0,0\right]^{T}$ and $\chi_{-}^{(\mathrm{N})}(r, \varphi)=\left[0,0,-i J_{m}\left(k_{-} r\right), J_{m+1}\left(k_{-} r\right) e^{i \varphi}\right]^{T}$ are the two eigenstates, and $k_{ \pm}=\left(E_{\mathrm{F}}^{(\mathrm{G})} \pm E\right) /\left(\hbar v_{\mathrm{F}}\right)$. In the superconducting region $r>R$ where the pair potential is $\Delta^{(G)}$ the wave function has the form $\Psi_{m}^{(\mathrm{S})}(r, \varphi)=\left(c_{+}^{(\mathrm{S})} \chi_{+}^{(\mathrm{S})}(r, \varphi)+c_{-}^{(\mathrm{S})} \chi_{-}^{(\mathrm{S})}(r, \varphi)\right) e^{i m \varphi}$, where $\chi_{+}^{(\mathrm{S})}(r, \varphi)=\left[u_{+}^{(\mathrm{S})}, v_{+}^{(\mathrm{S})}\right]^{T}, u_{+}^{(\mathrm{S})}=\gamma_{+} v_{+}^{(\mathrm{S})}$, $v_{+}^{(\mathrm{S})}=\left[-i H_{m}^{(1)}\left(q_{+} r\right), H_{m+1}^{(1)}\left(q_{+} r\right) e^{i \varphi}\right]^{T}$. The eigenstate $\chi_{-}^{(\mathrm{S})}(r, \varphi)$ is obtained by the replacement $+\rightarrow-$ and the first kind of Hankel functions to the second one and $q_{ \pm}=\left(E_{\mathrm{F}}^{(\mathrm{G})} \pm i \sqrt{\left[\Delta^{(G)}\right]^{2}-E^{2}}\right) /\left(\hbar v_{\mathrm{F}}\right)$, while $\gamma_{ \pm}=$ $e^{ \pm i \arccos \left(E / \Delta^{(G)}\right)}$. Here $J_{m}(x)$ and $H_{m}^{(1,2)}(x)$ are the Bessel and the Hankel functions [15]. To ensure that the wave function of the bound states is normalizable, the wave function in the superconducting region must go to zero as $r \rightarrow \infty$. This condition can be satisfied by choosing the appropriate Hankel function in the eigenstates $\chi_{ \pm}^{(\mathrm{S})}(r, \varphi)$ [15]. Finally, the unknown coefficients $c_{ \pm}^{(\mathrm{N})}$ and $c_{ \pm}^{(\mathrm{S})}$ can be determined from the boundary conditions $\Psi_{m}^{(\mathrm{N})}(r=R, \varphi)=\Psi_{m}^{(\mathrm{S})}(r=R, \varphi)$ valid for any $\varphi$. Thus, the condition for non-trivial solutions of the coefficients $c_{ \pm}^{(\mathrm{N})}$ and $c_{ \pm}^{(\mathrm{S})}$ can be found from the zeros of a four by four determinant. After some algebra we obtain a quite simple secular equation for the energy levels with fixed angular momentum index $m$ :

$$
\begin{array}{r}
\operatorname{Im}\left\{\gamma_{+} \mathrm{D}_{\mathrm{GS}}^{(+)}(\mathrm{m}, \mathrm{E}) \mathrm{D}_{\mathrm{GS}}^{(-)}(\mathrm{m}, \mathrm{E})\right\}=0, \\
D_{\mathrm{GS}}^{(+)}(m, E)=\left|\begin{array}{cc}
J_{m}\left(k_{+} R\right) & H_{m}^{(1)}\left(q_{+} R\right) \\
J_{m+1}\left(k_{+} R\right) & H_{m+1}^{(1)}\left(q_{+} R\right)
\end{array}\right|,
\end{array}
$$

and $D_{\mathrm{GS}}^{(-)}(m, E)=\left[D_{\mathrm{GS}}^{(+)}(m,-E)\right]^{*}$, and $\operatorname{Im}\{$.$\} and$ * stand for the imaginary part and the complex conjugation, respectively. Note that $H_{m}^{(2)}\left(q_{-} R\right)=$ $\left[H_{m}^{(1)}\left(q_{+} R\right)\right]^{*}$. The solutions of Eq. (1) for $m=$ $0, \pm 1, \pm 2, \cdots$, are the exact energy levels of a circular shape GAB. Note that Eq. (11) is valid both in the case of Andreev retro-reflection $\left(E_{\mathrm{F}}^{(\mathrm{G})}>\Delta^{(G)}>E\right)$ and for specular Andreev reflection $\left(E_{\mathrm{F}}^{(\mathrm{G})}<E<\Delta^{(G)}\right)$. One can also notice that the eigenenergies depend only on two dimensionless parameters: $E_{\mathrm{F}}^{(\mathrm{G})} / \Delta^{(G)}$ and $\xi_{c}^{(G)} / R$, where $\xi_{c}^{(G)}=\hbar v_{\mathrm{F}}^{(G)} / \Delta^{(G)}$ is the coherence length in the superconducting graphene.

We now compare the density of states (DOS) $\varrho(E)=$ $\sum_{n m} \delta\left(E-E_{n m}\right)$ of a circular shape GAB and of the corresponding NS billiard. For details of the calculation see a similar calculation for NS billiards in Ref. [16]. It is more convenient to plot the integrated DOS, namely the so-called step function $N(E)=\sum_{n m} \Theta\left(E-E_{n m}\right)$, where $\Theta(x)$ is the Heaviside function. Our numerical results for $E_{\mathrm{F}}^{(\mathrm{N})} / \Delta^{(N)}=E_{\mathrm{F}}^{(\mathrm{G})} /\left(2 \Delta^{(G)}\right)$ are shown in Fig. 1. One can see that the step functions for the considered NS billiard and $\mathrm{GAB}$ are indeed very similar. It is also clear from Fig. 1 that the DOS $\varrho(E)=d N(E) / d E$ shows singularities at certain energies $E_{n}^{(\text {sing })}$. Singularities of this kind arise in the case of NS billiards as well (see e.g. Ref. [16]) and we shall discuss their origin below. 


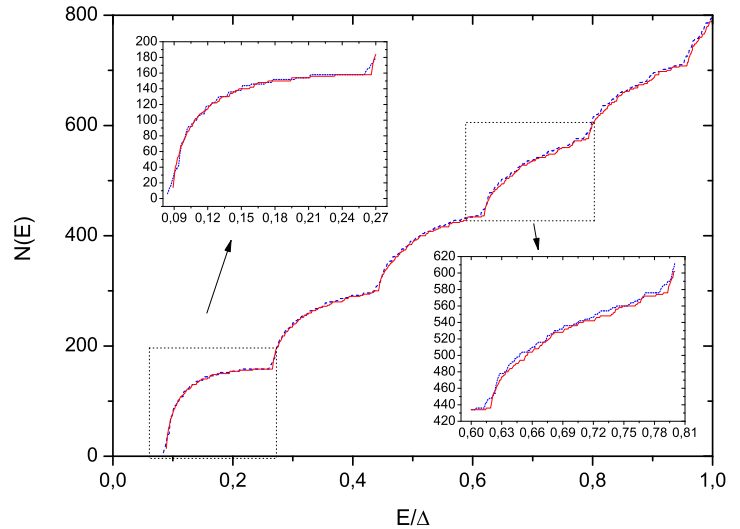

FIG. 1: (color online) The exact step function $N(E)$ for circular shape GAB (red line) and NS (blue line) billiards in the case of Andreev retro-reflection. The parameters for GAB and NS billiard are $E_{\mathrm{F}}^{(\mathrm{G})} / \Delta^{(G)}=10$ and $E_{\mathrm{F}}^{(\mathrm{N})} / \Delta^{(N)}=5$, respectively, and $\xi_{c}^{(G)} / R=0.12$ for both cases. The insets show the enlarged parts of the main frame. The trivial factor 2 owing to the valley degeneracy in graphene is not included.

We now demonstrate on the example of circular GABs that at semiclassical level the results for NS billiards and GABs can be mapped into each other by choosing the parameters appropriately. In numerous works [3, 17, 18, 19, 20, 21] it was shown that for NS billiards in semiclassical approximation the step function reads

$$
\begin{aligned}
N_{\mathrm{BS}}(E) & =M \sum_{n=0}^{\infty}\left\{1-F\left[s_{n}(E)\right]\right\} \\
s_{n}(E) & =\frac{n \pi+\arccos \left(E / \Delta^{(N)}\right)}{E / \Delta^{(N)}} \xi_{c}^{(N)} .
\end{aligned}
$$

Here $M$ is the number of open channels in the normal region, $\xi_{c}^{(N)}=\hbar v_{\mathrm{F}}^{(N)} / \Delta^{(N)}$ is the coherence length in the NS system, $F(s)=\int_{0}^{s} P\left(s^{\prime}\right) d s^{\prime}$ is the integrated path length distribution and $P(s)$ is the classical probability that an electron entering the billiard at the NS interface returns to the interface after a path of length $s$. The path length distribution $P(s)$ is normalized to one, i.e., $\int_{0}^{\infty} P(s) d s=1$ and one can see that it is a purely geometry-dependent function. In particular, for circular billiards it was found that $P(s)=\frac{1}{(2 R)^{2}} \frac{s}{\sqrt{1-(s / 2 R)^{2}}} \Theta(2 R-s)$ and $M=$ $2 \pi k_{\mathrm{F}}^{(N)} R$ [16]. Finally, the quantity $s_{n}(E)$ in Eq. (2b) depends on the quantization condition for the periodic motion of the electron-hole quasiparicles [17, 20]. As it has been pointed out in the introduction, in good approximation the quasiparticles have linear dispersion in the non-superconducting region for both GABs and NS billiards. If the effect of the superconductor in GABs can be taken into account by a simple phase shift $-\arccos \left(E / \Delta^{(G)}\right)$ [12], expressions of the type of Eq. (2) can be used to calculate the semiclassical approximation of $N(E)$ for GABs as well.
Moreover, employing the same steps as in Ref. [16], from Eq. (11) one can derive the following semiclassical quantization rule for circular shape GABs:

$$
\begin{aligned}
& S_{+}(E)-\mu_{r} S_{-}(E)-2 \arccos \frac{E}{\Delta^{(G)}}=2 \pi\left(n+\frac{1-\mu_{r}}{4}\right) \\
& S_{ \pm}(E)=2 \sqrt{\left(\left|k_{ \pm}\right| R\right)^{2}-m^{2}}-2|m| \arccos \frac{|m|}{\left|k_{ \pm}\right| R}
\end{aligned}
$$

where $\mu_{r}=1,-1$ for Andreev retro-reflection and specular Andreev reflection, respectively, and $n$ is a nonnegative integer. Functions $S_{ \pm}(E)$ are the radial action (in units of $\hbar$ ) of electrons and holes [22] and the term $-2 \arccos E / \Delta^{(G)}$ in Eq. (3a) accounts for the two Andreev reflections in one period of the orbit, while the second term in the left hand side of Eq. (3a) results from the sum and the difference of the Maslov indices $\pi / 4$ of the electron-like and hole-like particles for $\mu_{r}=1$ and $\mu_{r}=-1$, respectively.

Formally, in the case of Andreev retro-reflection the quantization condition shown in Eq. (3) is the same as for a circular NS billiard [16], but the meaning of $k_{ \pm}$ is different for the two systems (for NS billiards see eg. Ref. 16]). However, from Eq. (3) it is easy to find that if $R^{(N)} / \xi_{c}^{(N)}=R^{(G)} / \xi_{c}^{(G)}$ and $E_{F}^{(N)} / \Delta^{(N)}=E_{F}^{(G)} /\left(2 \Delta^{(G)}\right)$ then to first order in $E / \Delta^{(N, G)}$ the quantization condition for circular GABs and NS billiards is the same and the step function $N(E)$ is given by Eq. (2) with coherence length $\xi_{c}^{(G)}$. The exact and semiclassically calculated $N(E)$ are plotted in Fig. 2. The agreement between the two results is excellent. Moreover, from Eq. (2), we

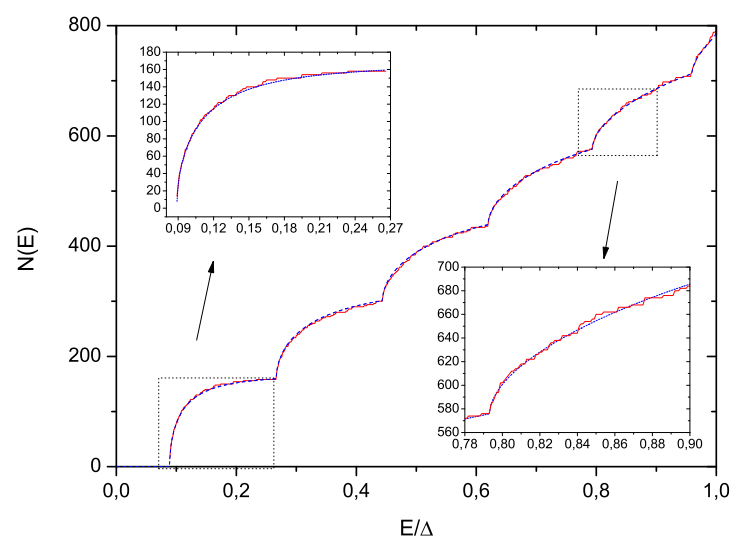

FIG. 2: (color online) The exact (red line) and the semiclassically (blue line) calculated step function $N(E)$ obtained from Eqs. (1) and (2), respectively for the case of Andreev retroreflection. The parameters are the same as in Fig. (11). The insets show the enlarged parts of the main frame.

find that the positions of the singularities in the DOS are given by $E_{n}^{(\operatorname{sing})} / \Delta^{(G)}=(n+1 / 2) \pi /\left(1+2 R / \xi_{c}^{(G)}\right)$ valid for such integers $n$ that $E_{n}^{(\operatorname{sing})}<\Delta^{(G)}$ holds. Note that the position $E_{n}^{(\text {sing })} / \Delta^{(G)}$ of the singularities depends only on $R / \xi_{c}^{(G)}$ but not on $E_{\mathrm{F}}^{(G)} / \Delta^{(G)}$. Therefore 
even if $E_{\mathrm{F}}^{(N)} / \Delta^{(N)} \neq E_{\mathrm{F}}^{(G)} /\left(2 \Delta^{(G)}\right)$ but $R^{(N)} / \xi_{c}^{(N)}=$ $R^{(G)} / \xi_{c}^{(G)}$, the singularities in the DOS for a circular GAB and NS billiard would appear at the same energies.

Next we consider the case of specular Andreev reflection in graphene Andreev billiards. Again, the solutions of Eqs. (11) and (3) give the exact and the semiclassically calculated energy levels of circular shape GABs. In Fig. 3 the calculated step function $N(E)$ is plotted and as one can see it is completely different from that obtained for the case of Andreev retro-reflection shown in Fig. 1. Moreover, the quantum results in Fig. 3 again show very

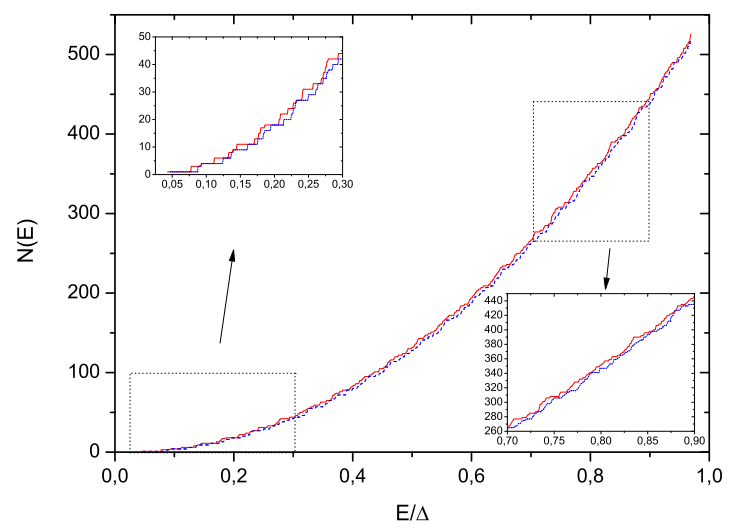

FIG. 3: (color online) The exact (red line) and the semiclassically (blue line) calculated step function $N(E)$ obtained from (1) and (3), respectively for specular Andreev reflection. The parameters are $E_{\mathrm{F}}^{(\mathrm{G})}=0$ and $\xi_{c}^{(G)} / R=0.03$. The insets show the enlarged parts of the main frame.

good agreement with the semiclassical ones that can be obtained from Eq. (3). This implies that in the case of specular Andreev reflection the DOS depends linearly on the energy for $E \rightarrow 0$. Namely, it can be shown from Eq. (3) that in this limit the DOS in semiclassical approximation (without the valley degeneracy) is given by $\rho(E)=8 \frac{E \mathcal{A}}{\pi^{3}\left(\hbar v_{F}\right)^{2}}$, where $\mathcal{A}$ is the area of the billiard. It is interesting to note therefore that (apart from the valley degeneracy) $\rho(E)$ is bigger by a factor of $16 / \pi^{2}$ than in the case of neutrino billiards [23].

In summary, we calculated the energy levels of graphene based Andreev billiards. We showed that for energy levels corresponding to the case of Andreev retroreflection the graphene based Andreev billiards in a very good approximation can be mapped to the normal metalsuperconducting billiards with the same geometry. We also derived a semiclassical quantization rule in graphene based Andreev billiards and the spectrum obtained from this rule agrees very well with that obtained from the exact quantum calculations for circular shape of GS billiards.

This work is supported by the Hungarian Science Foundation OTKA under the contracts No. T48782 and 75529. A. K. is supported partly by European Commission Contract No. MRTN-CT-2003-504574 and by EP-
SRC. We gratefully acknowledge fruitful discussions with C. W. J. Beenakker and V. Falko.

[1] A. F. Andreev, Zh. Eksp. Teor. Fiz. 46, 1823 (1964), [Sov. Phys. JETP, 19, 1228 (1964)].

[2] I. Kosztin, D. L. Maslov, and P. M. Goldbart, Phys. Rev. Lett. 75, 1735 (1995).

[3] A. Lodder and Y. V. Nazarov, Phys. Rev. B 58, 5783 (1998).

[4] C. W. J. Beenakker, Lecture Notes in Physics 667, 131 (2005).

[5] K. Novoselov et al., Science 306, 666 (2004).

[6] Y. Zhang, Y. Tan, H. Stormer, and P. Kim, Nature 438, 201 (2005).

[7] V. I. Fal'ko et al., Solid State Commun. 143, 33 (2007).

[8] P. Carmier and D. Ullmo, Phys. Rev. B 77, 245413 (2008); A. Kormányos, P. Rakyta, L. Oroszlány, and J. Cserti, Phys. Rev. B 78, 045430 (2008).

[9] Special issue of Solid State Commun. 143, June (2007); Eur. Phys. J. Special Topics 148, September (2007); M. I. Katsnelson, Materials Today 10, 20 (2007); M. I. Katsnelson and K. S. Novoselov, Solid State Commun. 143, 3 (2007); A. K. Geim and K. S. Novoselov, Nature Mat. 6, 183 (2007); A. H. C. Neto, F. Guinea, N. M. Peres, K. S. Novoselov, and A. K. Geim, Rev. Mod. Phys. 81, 109 (2009).

[10] C. W. J. Beenakker, Phys. Rev. Lett. 97, 067007 (2006).

[11] M. Titov and C. W. J. Beenakker, Phys. Rev. B 74, 041401(R) (2006); A. G. Moghaddam and M. Zareyan, Phys. Rev. B 74, 241403(R) (2006); M. Titov, A. Ossipov, and C. W. J. Beenakker, Phys. Rev. B 75, 045417 (2007); A. Ossipov, M. Titov, and C. W. J. Beenakker, Phys. Rev. B 75, 241401(R) (2007); D. Greenbaum, S. Das, G. Schwiete, and P. G. Silvestrov, Phys. Rev. B 75, 195437 (2007); C. W. J. Beenakker, A. R. Akhmerov, P. Recher, and J. Tworzydlo, Phys. Rev. B 77, 075409 (2008); P. Burset, A. Levy Yeyati, and A. Martín-Rodero, Phys. Rev. B 77, 205425 (2008); J. Linder and A. Sudbo, Phys. Rev. B 77, 064507 (2008).

[12] C. W. J. Beenakker, Rev. Mod. Phys. 80, 1337 (2008).

[13] H. B. Heersche et al., Nature 446, 56 (2007).

[14] F. Miao et al., Science 317, 1530 (2007).

[15] M. Abramowitz and I. A. Stegun, Handbook of Mathematical Functions, 9th ed. (Dover Publication, Inc., New York, 1972).

[16] J. Cserti, B. Béri, A. Kormányos, P. Pollner, and Z. Kaufmann, J. Phys.: Condens. Matter 16, 6737 (2004).

[17] J. A. Melsen, P. W. Brouwer, K. M. Frahm, and C. W. J. Beenakker, Europhys. Phys. Lett. 35, 7 (1996).

[18] H. Schomerus and C. W. J. Beenakker, Phys. Rev. Lett. 82, 2951 (1999).

[19] W. Ihra, M. Leadbeater, J. L. Vega, and K. Richter, Eur. Phys. J. B 21, 425 (2001).

[20] I. Adagideli and P. M. Goldbart, Int. J. Mod. Phys. B 16, 1381 (2002).

[21] J. Cserti, A. Kormányos, Z. Kaufmann, J. Koltai, and C. J. Lambert, Phys. Rev. Lett. 89, 057001 (2002); A. Kormányos, Z. Kaufmann, J. Cserti, and C. J. Lambert, Phys. Rev. B 67, 172506 (2003); F. Libisch, S. Rotter, J. Burgdörfer, A. Kormányos, and J. Cserti, Phys. Rev. B 
72, 075304 (2005).

[22] M. Brack and R. K. Bhaduri, in Semiclassical Physics, edited by D. Pines (Addison-Wesley Pub. Co., Inc., Amsterdam, The Netherlands, 1997).
[23] M. V. Berry and R. J. Mondragon, Proc. R. Soc. Lond. A 412, 53 (1987). 